Bundesgesundheitsbl 2020 · 63:1161-1167 https://doi.org/10.1007/s00103-020-03206-8 Online publiziert: 20. August 2020

(C) Der/die Autor(en) 2020

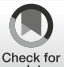

Jörg Schmidtke ${ }^{1,3}$ • Jörg T. Epplen ${ }^{2,3} \cdot$ Ralf Glaubitz $^{3}$. Tiemo Grimm ${ }^{4}$ • R. Peter Nippert ${ }^{5} \cdot$ Holger Tönnies $^{6} \cdot$ Klaus Zerres $^{7} \cdot$ Irmgard Nippert $^{8}$ ${ }^{1}$ Institut für Humangenetik, Medizinische Hochschule Hannover, Hannover, Deutschland ${ }^{2}$ Ruhr-Universität Bochum, Bochum, Deutschland ${ }^{3}$ amedes-genetics, Hannover, Deutschland

${ }^{4}$ Institut für Humangenetik, Biozentrum, Universität Würzburg, Würzburg, Deutschland

${ }^{5}$ Medizinische Fakultät, WWU Münster, Münster, Deutschland

${ }^{6}$ Geschäftsstelle Gendiagnostik-Kommission, Robert Koch-Institut, Berlin, Deutschland ${ }^{7}$ Institut für Humangenetik, RWTH Aachen, Aachen, Deutschland

${ }^{8}$ Institut für Humangenetik, Universitätsklinikum Münster, Münster, Deutschland

\title{
Humangenetische Beratung in Deutschland: Entwicklung der Inanspruchnahme
}

In keinen anderen Bereich der Medizin hat der Gesetzgeber in ähnlicher Weise unmittelbar eingegriffen wie in den der Genetik. Dies gilt nicht nur für Deutschland. In zahlreichen europäischen Ländern wurde ähnlich verfahren [2, 3]. Offenbar besteht - auch international - ein gesellschaftliches Interesse, genetischen Daten in der Medizin eine Sonderstellung zuzuweisen („genetischer Exzeptionalismus"; [4]) und den spezifischen Chancen und Risiken der Anwendung auf Gesetzesebene - und nicht nur auf der Basis der ärztlichen Selbstverwaltung - gerecht zu werden. Die hier vorgelegten Daten zur Inanspruchnahme der humangenetischen Beratung in den Jahren vor und nach dem Inkrafttreten des GenDG dienen einer informierten Abschätzung möglicher Auswirkungen des Gesetzes.

Zur Entwicklung der Inanspruchnahme genetischer Beratung in Deutschland lagen veröffentlichte Daten bisher nur für den Zeitraum 1996 bis 2004 vor [5, 6]. In diesem Zeitraum blieb die pro Jahr erbrachte Zahl von genetischen Beratungen, die von der gesetzlichen Krankenversicherung (GKV) vergütet wurden, im Wesentlichen konstant bei jährlich rund 47.000. Gleichzeitig stieg in diesem Zeitraum die Zahl der erbrachten diagnostischen genetischen Untersuchungen erheblich an, sodass sich die Schere zwi- schen Diagnostikleistungen und Beratungen immer weiter öffnete.

Die weitere Entwicklung der Zahl genetischer Beratungsleistungen in den Jahren unmittelbar vor und insbesondere nach Inkrafttreten des GenDG und der Umsetzung der Richtlinie der GEKO zur genetischen Beratung wurde bisher nicht erfasst.

Aufgrund der veralteten Datenlage konnte die Entwicklung des Umfangs genetischer Beratungen seit der Einführung des GenDG bisher nicht beschrieben werden.

Parallel zu der vom Bundesministerium für Gesundheit geförderten Studie "Genetische Beratung in Deutschland Struktur und Inanspruchnahme" (GenBIn-Studie; [7]) haben wir aktuelle Daten erhoben, die

1. die Entwicklung des Umfangs genetischer Beratungsleistungen sowohl vor als auch nach Inkrafttreten des GenDG dokumentieren und

2. zur Diskussion der beobachtbaren Entwicklung der Inanspruchnahme genetischer Beratung und der möglichen zukünftigen Entwicklung beitragen.

\section{Material und Methoden}

Über einen Zeitraum von 9 Jahren (2009 bis 2017) wurden die im Rahmen der (fachgebundene genetische Beratung). 


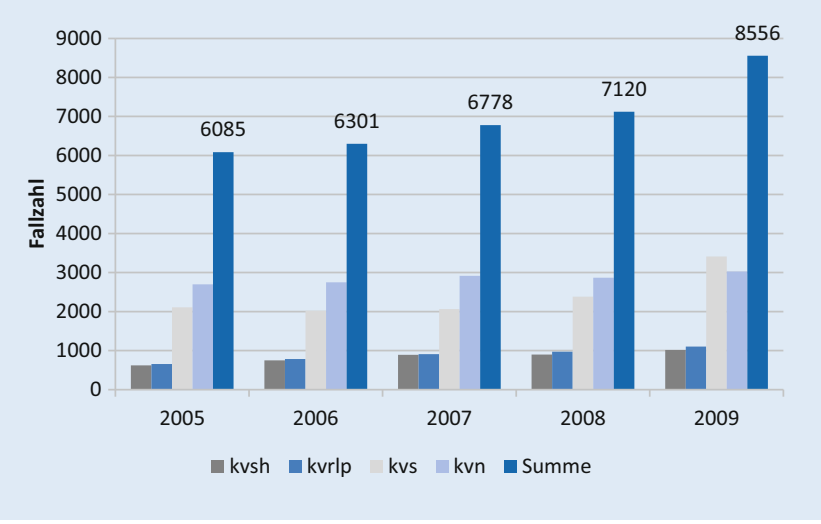

Abb. 1 A Genetische Beratungsleistungen 2005 bis 2009: Vergütung durch die gesetzliche Krankenversicherung (GKV) in 4 Einzel-KVen (Kassenärztlichen Vereinigungen). (Quelle: KV Schleswig-Holstein (kvsh), KV Rheinland-Pfalz (kvrlp), KV Sachsen (kvs), KV Niedersachsen (kvn))

gesetzlichen Krankenversicherung erbrachten und nach Einheitlichem Bewertungsmaßstab (EBM) über die kassenärztlichen Vereinigungen abrechenbaren humangenetischen Beratungsleistungen über eine Datenbankabfrage beim Zentralinstitut der Kassenärztlichen Bundesvereinigung (ZI-KBV) erfasst und dokumentiert.

Die Daten der Jahre 2005 bis 2008 waren zum Zeitpunkt des Beginns der Abfrage (Juli 2016) nicht mehr aus der ZI-KBV-Datenbank abrufbar. Es wurden daher alle Kassenärztlichen Vereinigungen (KV) Deutschlands um die Übermittlung etwaiger noch vorhandener Daten für diesen Zeitraum gebeten.

Bedingt durch die sich wandelnde Struktur der ZI-KBV-Datenbank standen für den veröffentlichten Zeitraum 1996-2004 genetische Beratungsleistungen aller Fachgruppen zur Verfügung, für den Zeitraum 2009 bis 2017 konnten nur noch die Beratungsleistungen von Pädiatern, Gynäkologen und $\mathrm{Hu}-$ mangenetikern (die zusammen aber den weitaus größten Anteil erbringen) präzise recherchiert werden.

Die Abfragen wurden 2016 parallel zu der GenBIn-Studie begonnen und 2019 abgeschlossen.

Die aktuell nicht abrechenbaren fachgebundenen genetischen Beratungen [1] werden mit dieser Abfrage nicht erfasst.

Eine fachgebundene genetische Beratung erfolgt durch qualifizierte Fachärztinnen und Fachärzte im Hinblick auf fachspezifische Fragestellungen, die im
Rahmen des jeweiligen ärztlichen Fachgebiets anfallen. Sie erfordert daher keine übergreifende, die Fachgrenzen überschreitende genetische Expertise. Ergeben sich in der genetischen Beratung Hinweise auf übergeordnete Fragestellungen, die über die eigenen Fachgrenzen hinausgehen, soll laut Richtlinie der GEKO [1] eine Überweisung zur Fachärztin oder zum Facharzt für Humangenetik oder zu einer/einem auf dem Fachgebiet entsprechend qualifizierten Ärztin oder Arzt mit Zusatzbezeichnung „Medizinische Genetik“ erfolgen.

Ebenso nicht erfasst werden konnten genetische Beratungen im Rahmen pauschalierter Abrechnungen sowie für die private Krankenversicherung und Selbstzahler, die zusammengenommen jedoch nur einen geringen Anteil am Beratungsaufkommen darstellen.

Für die Diskussion der beobachtbaren Entwicklung der Inanspruchnahme genetischer Beratung und der möglichen zukünftigen Entwicklung werden zusätzlich Daten der GenBIn-Datenbank herangezogen. Die GenBIn-Datenbank umfasst Daten von 5256 genetischen Beratungen aus den Jahren $2011(n=2091)$ und 2016/2017 $(n=3165)$. Das Jahr 2011 wurde bewusst gewählt, um den Status quo ante vor dem 01.02.2012 zu dokumentieren. Ab dem 01.02.2012 sind nur noch solche Ärztinnen und Ärzte zur fachgebundenen genetischen Beratung qualifiziert, die die in der Richtlinie der GEKO zur Qualifikation zur gene- tischen Beratung festgelegte Beratungsqualifikation erworben haben.

Initial ist die GenBIn-Datenbank aus der bundesweiten Kooperation von 27 genetischen Beratungseinrichtungen entstanden (13 universitäre Einrichtungen, 1 akademisches Lehrkrankenhaus, 13 Beratungseinrichtungen in der Niederlassung). Diese Einrichtungen erklärten sich 2013 bereit, retrospektiv für 2011 Falldaten zur genetischen Beratung für die Datenbank nach einem standardisierten Verfahren (25 Fälle pro Quartal 2011) aus ihren Archiven zu ziehen und zu dokumentieren. Bezogen auf das Erfassungsjahr 2011 repräsentieren diese Einrichtungen knapp ein Viertel der damaligen Beratungsstellen $(n=111)$. An der nachfolgenden Dokumentation für 2016/2017 nahmen insgesamt 34 Beratungseinrichtungen teil (19 universitäre Einrichtungen, 2 akademische Lehrkrankenhäuser, 13 Beratungseinrichtungen in der Niederlassung). Eine Übersicht über die geografische Verteilung in 2016/2017 findet sich im GenBInForschungsbericht [7]. Von den 27 Einrichtungen, die die Beratungsfälle für 2011 übermittelten, sind bedingt durch Schließungen (2), mangelnde personelle Kapazitäten (1) und Änderungen in der Leitungsebene (1) noch 23 in der Dokumentation für 2016/2017 vertreten. Neu hinzu kamen 11 Einrichtungen, darunter 2, die den durch Schließung bedingten Ausfall der 2 Einrichtungen in derselben Region kompensierten.

Die standardisierten Falldokumentationen der GenBIn-Datenbank erlauben erstmals eine systematische Beschreibung der Erbringung und Nutzung genetischer Beratungsleistungen und deren Vergleich innerhalb verschiedener Zeiträume in einem Kollektiv unterschiedlicher Beratungseinrichtungen. Damit kann die GenBIn-Datenbank als Grundlage für ein systematisches Monitoring der Entwicklung der Beratungspraxis nach dem Inkrafttreten des GenDG und der Umsetzung der Richtlinie der GEKO genutzt werden [1]. Hierzu gab es bisher keine Daten.

Eine ausführliche Beschreibung der Methoden der GenBIn-Studie, der Datenerhebung sowie der teilnehmenden humangenetischen Beratungseinrich- 
Bundesgesundheitsbl 2020 • 63:1161-1167 https://doi.org/10.1007/s00103-020-03206-8

(c) Der/die Autor(en) 2020

\section{J. Schmidtke · J. T. Epplen · R. Glaubitz · T. Grimm • R. P. Nippert · H. Tönnies · K. Zerres · I. Nippert}

\section{Humangenetische Beratung in Deutschland: Entwicklung der Inanspruchnahme}

\section{Zusammenfassung}

Hintergrund. Mit dem Gendiagnostikgesetz (GenDG) hat der deutsche Gesetzgeber tief greifende Vorschriften für die humangenetische Leistungserbringung einschließlich der genetischen Beratung erlassen. In dieser Arbeit werden Daten zur Inanspruchnahme der humangenetischen Beratung in den Jahren vor und nach dem Inkrafttreten des GenDG vorgelegt. Diese Daten sollen einer informierten Abschätzung möglicher Auswirkungen des Gesetzes dienen.

Material und Methoden. Die in den Jahren 2005 bis 2017 im Rahmen der gesetzlichen Krankenversicherung erbrachten und nach Einheitlichem Bewertungsmaßstab (EBM) über die kassenärztlichen Vereinigungen (KVen) abrechenbaren humangenetischen Beratungsleistungen wurden über eine
Datenbankabfrage beim Zentralinstitut der Kassenärztlichen Bundesvereinigung (ZIKBV) sowie bei einzelnen KVen Deutschlands erfasst. Für die Diskussion der beobachtbaren Entwicklung der Inanspruchnahme genetischer Beratung und der möglichen zukünftigen Entwicklung werden zusätzlich Daten zum Überweisungsverhalten, den Wartezeiten und Beratungsanlässen aus der GenBIn(Studie "Genetische Beratung in Deutschland")-Datenbank herangezogen. Ergebnisse und Diskussion. Die Inanspruchnahme der genetischen Beratung wächst seit 2009 mit einer durchschnittlichen Rate von ca. $6 \%$ pro Jahr. Die gestiegene Nachfrage wird begleitet von veränderten relativen Anteilen der Zuweiser und steigenden Selbstüberweisungen und hat zu längeren
Wartezeiten geführt. Unsere Daten legen die Vermutung nahe, dass der beobachtbare ständige Anstieg genetischer Beratungsleistungen multifaktoriell bedingt ist und nicht allein auf das GenDG zurückzuführen ist. Da die Zahl der hier wesentlichen Leistungserbringer, der vertragsärztlichen Fachärzte für Humangenetik, nahezu konstant bleibt, ist absehbar, dass bei weiter steigender Inanspruchnahme Kapazitätsgrenzen erreicht werden.

\section{Schlüsselwörter}

Gendiagnostikgesetz - GendiagnostikKommission - GenBIn(Studie "Genetische Beratung in Deutschland")-Datenbank · ZIKBV(Zentralinstitut der Kassenärztlichen Bundesvereinigung)-Datenbank

\section{Genetic counseling in Germany: development of demand}

\section{Abstract}

Background. With the Act on Genetic Testing (GenDG), the German legislator has issued far-reaching regulations for human genetic services, including genetic counseling. This paper presents data on the use of human genetic counseling in the years before and after the entry into force of GenDG in order to provide an informed assessment of the possible effects of the law.

Materials and Methods. Over a period of 13 years (2005 to 2017), the human genetic counseling services provided within the framework of the statutory health insurance and billable by EBM via the Kassenärztliche associations were recorded via a database query at the Central Institute of the National Association of Statutory Health Insurance
Physicians (ZI-KBV) and via individual Kassenärztliche Vereinigungen Deutschlands. For the discussion of the observable development of using genetic counseling and possible future development, additional data on the referral behavior, the waiting times, processing time, and reasons for consultations were extracted from the GenBIn database.

Results and Discussion. Demand for genetic counseling has steadily increased at an average rate of approximately $6 \%$ per year since 2009. This increase started well before the enactment of the GenDG and may be attributed to a multiplicity of factors. Change in demand for genetic counseling is characterized by increasing self-referrals and by increasing referrals by specialists other than obstetricians/gynecologists. Waiting times between 2011 and 2016/2017 have increased. While demand has been growing, the number of key service providers, the contracted medical specialists in human genetics, has remained almost constant. It is foreseeable that capacity limits will be reached if both trends continue.

\section{Keywords}

German Act on Genetic Testing · German Commission on Genetic Testing - GenBIn (Study "Genetische Beratung in Deutschland") database - ZI-KBV (Central Institute of the National Association of the Statutory Health Insurance Physicians) database tungen wurde im GenBIn-Forschungsbericht vom Robert Koch-Institut (RKI) in 2019 publiziert (siehe oben).

\section{Ergebnisse}

Die Entwicklung der Inanspruchnahme der genetischen Beratung 2005 bis 2017

\section{5 bis 2009 Entwicklung in 4 Einzel-KVen}

4 Einzel-KVen, Schleswig-Holstein, Rheinland-Pfalz, Sachsen und Niedersachsen, die einen Bevölkerungsanteil in Deutschland von zusammen $23 \%$ repräsentieren, stellten ihre Daten für diesen
Zeitraum zur Verfügung. Aufgrund der unterschiedlichen Dokumentation von Fachgruppen konnte für die $4 \mathrm{KVen}$ gemeinsam nur die Zahl von genetischen Beratungen summiert über alle Fachgruppen erfasst werden (• Abb. 1).

Die Daten der 4 KVen zeigen, dass die Anzahl der vergüteten Beratungen von 2005 bis 2008 jährlich anstieg (• Abb. 1). Insgesamt nahm sie im Zeitraum von 4 Jahren um $17 \%$ zu. Im Zeitraum 2008 bis 2009 stieg die Zahl der Beratungen dann besonders stark an: Innerhalb eines Jah- 


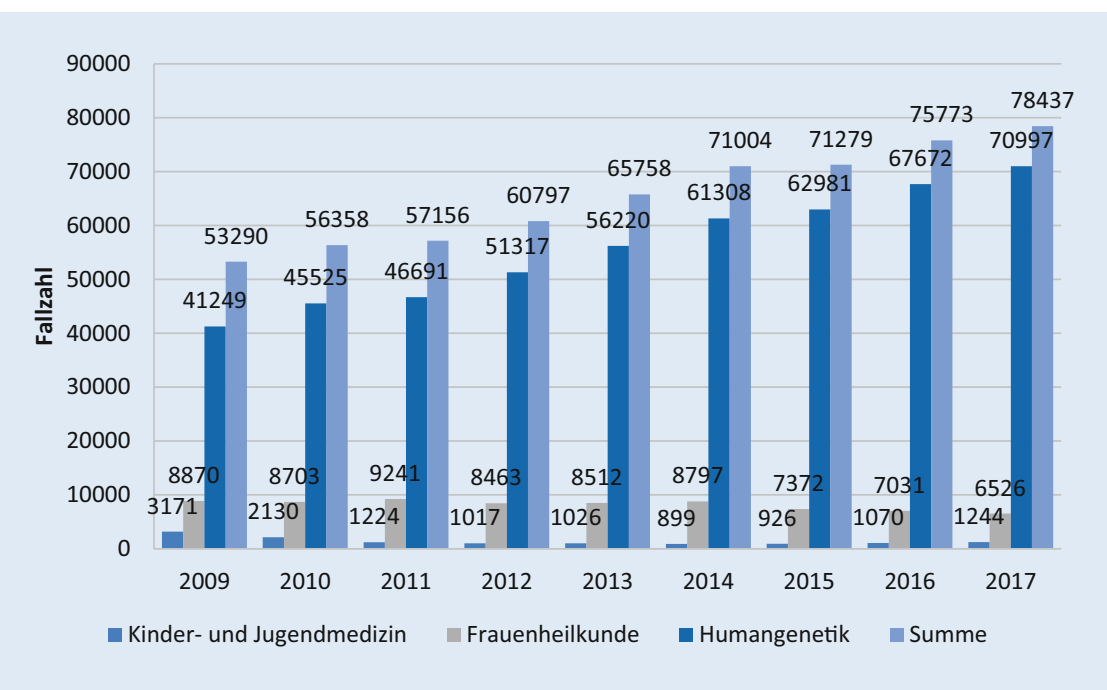

Abb. 2 A Genetische Beratungsleistungen 2009 bis 2017: Vergütung durch die gesetzliche Krankenversicherung (GKV), alle KVen (Kassenärztliche Vereinigungen). (Quelle: ZI-KBV[Zentralinstitut der Kassenärztlichen Bundesvereinigung]-Datenbank)

res erhöhte sie sich um $20 \%$. Insgesamt stiegen die Beratungszahlen im Zeitraum 2005 bis 2009 um 40,6\%. Es ist nicht auszuschließen, dass der im Vergleich zu den Vorjahren starke Anstieg in 2009 von der öffentlichen Diskussion des GenDG besonders beeinflusst wurde (GenDGAusfertigungsdatum am 31.07.2009; Inkrafttreten am 01.02.2010).

\section{Bundesweite Entwicklung 2009 bis 2017}

Seit dem Beschlussjahr des GenDG (2009) ist die Zahl genetischer Beratungsleistungen, die von der GKV bis Ende 2017 vergütet wurden, um fast $50 \%(47,3 \%)$ gestiegen, seit dem Inkrafttreten des GenDG in 2010 um $39 \%$ (2010: 56.358 Beratungen, 2017: 78.437; - Abb. 2). Die Anzahl der Beratungen, die davon von Fachärztinnen und Fachärzten für Humangenetik erbracht wurden, ist seit $2009 \mathrm{um}$ mehr als $72 \%$ gestiegen (2009: 41.249, 2017: 70.997), seit 2010 um $56 \%$ (• Abb. 2). Bei der Bewertung dieses Anstiegs ist $\mathrm{zu}$ beachten, dass seit dem 01.10.2015 die genetische Beratung nur noch von Fachärztinnen und Fachärzten für $\mathrm{Hu}$ mangenetik und von Fachärztinnen und Fachärzten mit der Zusatzbezeichnung „Medizinische Genetik“ in der GKV abgerechnet werden kann.

Insgesamt sind kontinuierlich jährliche Steigerungsraten im Zeitraum 2009 bis 2017 zu beobachten. Werden die Daten der 4 Einzel-KVen hinzugezogen, ist davon auszugehen, dass schon seit 2005 die Zahl der durch die GKV vergüteten genetischen Beratungen jährlich kontinuierlich steigt.

\section{Die Inanspruchnahme der genetischen Beratung in 2016/2017 im Vergleich zu 2011}

Der Vergleich der Inanspruchnahme der genetischen Beratung in 2011 (Status quo ante s. oben) mit der Inanspruchnahme in 2017 (für GenBIn-Daten: 2016/2017) dokumentiert Veränderungen in folgenden Bereichen.

Anzahl der Überweisungen. Die genetischen Beratungsfälle, die von der GKV insgesamt vergütet werden, sind seit 2011 von 57.156 auf $78.437(+37,2 \%)$ in 2017 gestiegen. Die von Fachärztinnen und Fachärzten für Humangenetik erbrachten Beratungen stiegen im gleichen Zeitraum von 46.691 auf 70.997 (+52,1\%). Die Humangenetik hat somit in diesem Zeitraum ihren Anteil an der Leistungserbringung deutlich gesteigert.

Spektrum der Überweiser (GenBIn-Datenbank). Die genetischen Beratungseinrichtungen erhielten in 2016/2017 wie in 2011 ihre häufigsten Zuweisungen von Fachärztinnen und Fachärzten für Frauenheilkunde und Geburtshil- fe (• Abb. 3a, b). Mit 34\% waren die Anteile in den Einrichtungen an den Universitäten und akademischen Lehrkrankenhäusern (ALK) deutlich geringer als die Anteile in der Niederlassung (63\%). In der Niederlassung kamen nur $37 \%$ der genetischen Beratungsfälle von anderen Zuweisern. Den größten Anteil erhielten hier mit $13 \%$ Fachärztinnen und Fachärzte für Kinder- und Jugendmedizin und Selbstüberweiser (10\%). Die Beratungseinrichtungen an den Universitäten und ALK erhielten dagegen $66 \%$ ihrer Zuweisungen von anderen Fachärztinnen und Fachärzten, insbesondere von jenen für Allgemeinmedizin (20\%), innere Medizin (12\%), Kinder- und Jugendmedizin (12\%) und anderen Fachärztinnen und Fachärzten (12\%). Der Anteil von Selbstüberweisungen entsprach mit $10 \%$ demjenigen der Beratungseinrichtungen in der Niederlassung.

Deutlich verändert haben sich die relativen Anteile der Zuweiser zu den genetischen Beratungseinrichtungen in der Niederlassung in 2016/2017 verglichen mit den Anteilen in 2011. Dominierten 2011 die Zuweisungen der Fachärztinnen und Fachärzte für Frauenheilkunde und Geburtshilfe mit einem Anteil von $83 \%$ von allen erhaltenen Zuweisungen, so sind diese Zuweisungen 2016/2017 mit einem Anteil von 63\%, um 20 Prozentpunkte geringer. Der Anteil der Überweisungen durch andere Fachärztinnen und Fachärzte und von Selbstüberweisungen hat sich mehr als verdoppelt. Betrug er in 2011 insgesamt $17 \%$, waren es in $2016 / 2017$ bereits $37 \%$. Insbesondere gestiegen sind die Anteile von Überweisungen von Fachärztinnen und Fachärzten für Kinder- und Jugendmedizin mit $13 \%$ (4\% in 2011) und der Anteil von Selbstüberweisungen mit $10 \%$ (2\% in 2011).

Wartezeiten (GenBIn-Datenbank). Die Wartezeiten zwischen der Anmeldung zur Beratung und dem Tag der ersten Beratung haben sich 2016/2017 erhöht. Der Median der Wartezeit ist um 6 Tage gestiegen: 2011 betrug die mittlere Wartezeit 41,18 Tage (Median 25 Tage), 2016/2017 betrug sie 47,14 Tage (Median 31 Tage; $\bullet$ Abb. 4). 


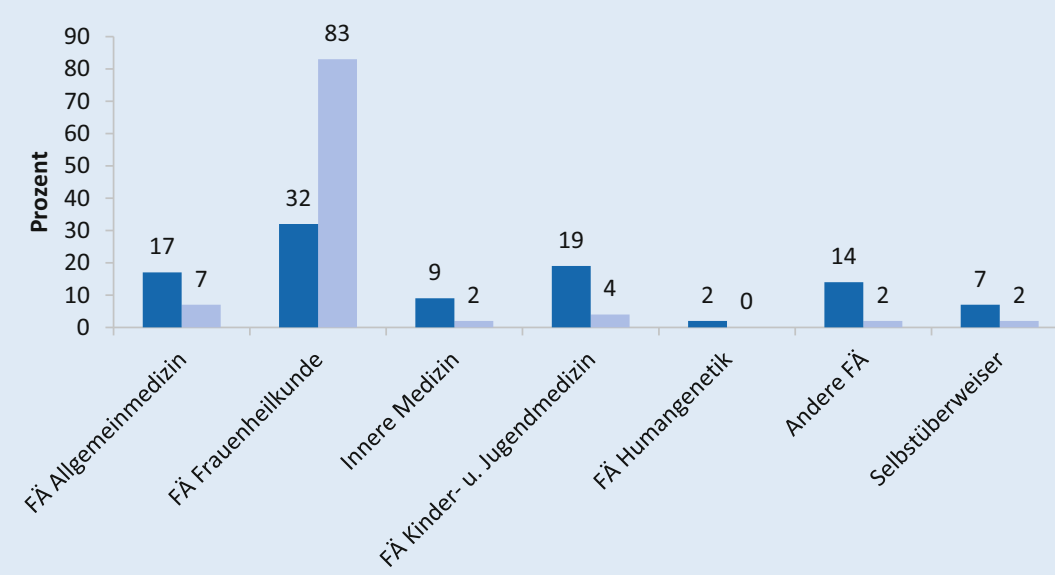

a

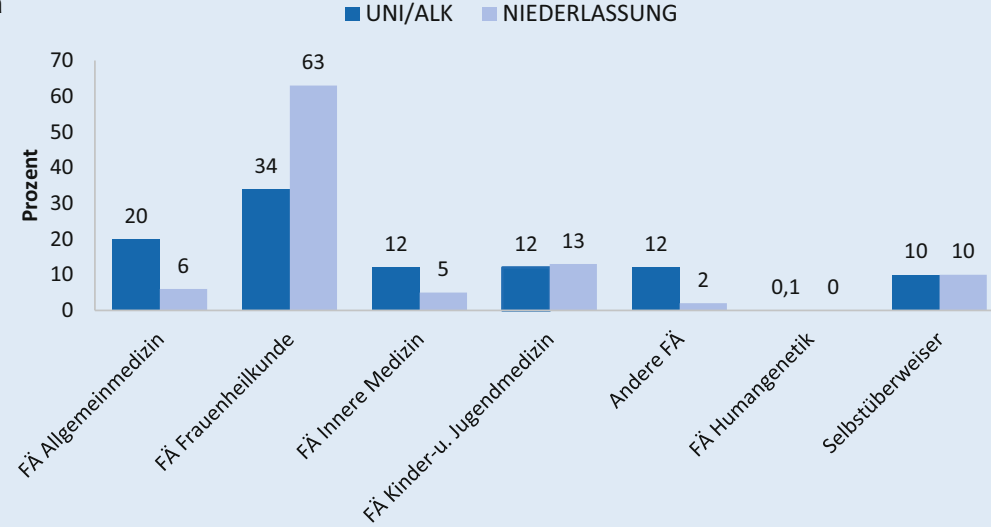

b

- UNI/ALK NIEDERLASSUNG bis unter $2 \mathrm{~h}$ berichtet (-Abb. 5 ). In den universitären Einrichtungen und den Einrichtungen der ALK traf dieser Zeitaufwand auf $35 \%$ der Beratungsfälle zu. $65 \%$ der Beratungsfälle erforderten hier einen Zeitaufwand von 2 und mehr Stunden.

\section{Beratungsanlässe in 2016/2017 (GenBIn-Datenbank)}

In 2016/2017 erhielt die Mehrheit (55\%) der dokumentierten Beratungsfälle eine humangenetische Beratung im Rahmen einer diagnostischen genetischen Untersuchung außerhalb einer Schwangerschaft. Hier fand die humangenetische Beratung am häufigsten (59\%) vor einer genetischen Untersuchung statt. Der Anteil von Beratungsfällen, die vor einer Untersuchung und zu Befundergebnissen beraten wurden, ist deutlich geringer $(28 \%)$. Nur zu einem Befundergebnis wurden $13 \%$ der Fälle beraten. $25 \%$ aller Beratungen fanden im Kontext einer prädiktiven genetischen Untersuchung einer nicht manifest erkrankten Rat suchenden Person (keine bestehende Schwangerschaft) statt. $63 \%$ dieser Beratungen fanden nur vor einer Untersuchung statt. $37 \%$ der Fälle wurden entweder nur zu einem Befundergebnis (5\%) oder vor und nach dem Vorliegen des Untersuchungsergebnisses (32\%) beraten.

Humangenetische Beratungen nach einem Direct-to-consumer(DTC)-Gentest (DTC-Gentests werden in der Regel ohne direkte ärztliche Indikationsstellung außerhalb des medizinischen Umfelds angeboten und erworben) oder einer pharmakogenetischen Untersuchung waren mit einem Anteil von $<0,1 \%$ in $2016 / 2017$ sehr selten.

Humangenetische Beratung im Kontext vorgeburtlichergenetischer Untersuchungsmöglichkeiten und Risikoabklärung fand 2016/2017 vor allem in der Niederlassung statt, weniger in den universitären Einrichtungen. In den Einrichtungen in der Niederlassung hatten diese Beratungen einen Anteil von $23 \%$, an den universitären Einrichtungen hatten sie einen Anteil von $9 \%$ an allen Beratungen.

Die Beratungsfälle der GenBIn-Datenbank für 2016/2017 vor der nichtinvasiven Pränataldiagnostik (NIPD) zur

\section{Zeitaufwand für die genetische}

wand $(=$ Dauer des direkten Patienten beit) dokumentiert werden. Da für 2011 die Daten retrospektiv erhoben wurden, fehlen hierzu häufig Angaben [7]. In 2016/2017 betrug der mittlere Zeitaufwand für einen Beratungsfall in den universitären und ALK-Einrichtungen 5,71 halbe Stunden (Median: 5,28). In den Einrichtungen in der Niederlassung war der Zeitaufwand etwas niedriger. Er betrug im Mittel 5,0 (Median: 4,6). In volle Stunden umgerechnet, wurde für $51 \%$ der Beratungsfälle in den Niederlassungen ein Zeitaufwand von maximal 1,5 


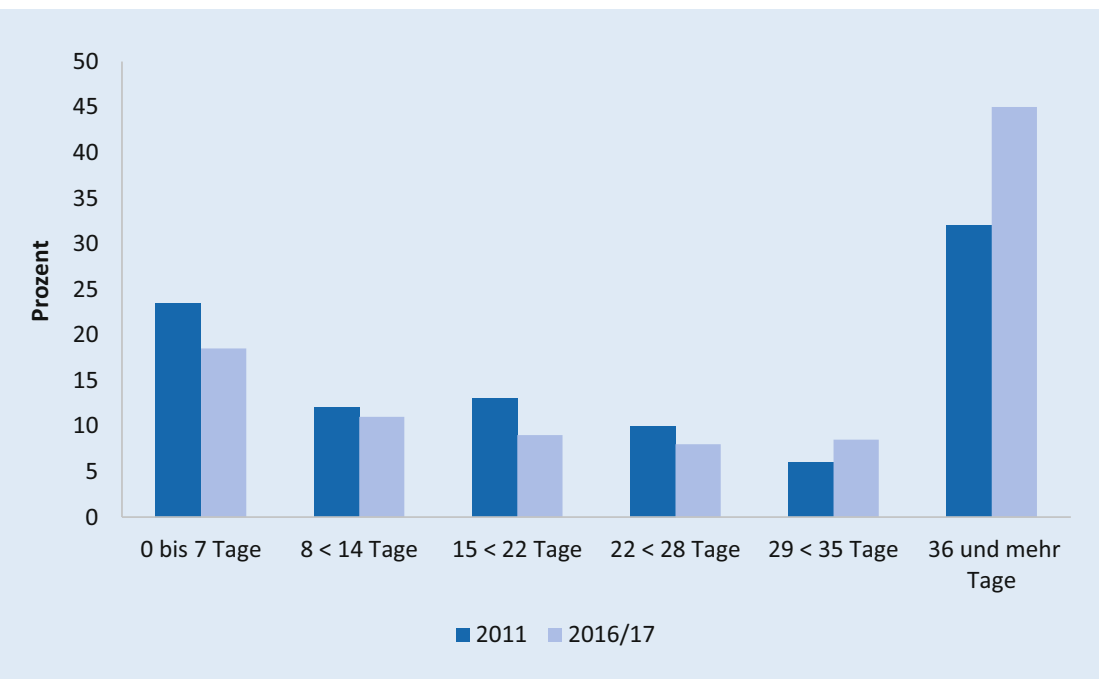

Abb. 4 \ Wartezeit zwischen Datum der Anmeldung zur Beratung und Datum der 1. Beratungssitzung, Vergleich GenBIn (2011) und GenBIn (2016/2017; \%). GenBIn 2011: gültige Fälle 1191, fehlende Fälle 900; Mittelwert 41,18 Tage; Median 25,00 Tage; Std.-Abweichung 49,348 Tage; Minimum 0 Tage; Maximum 461 Tage; GenBIn 2016/2017: gültige Fälle 3120, fehlende Fälle 45; Mittelwert 47,14 Tage, Median 31,00 Tage; Std.-Abweichung 51,766 Tage; Minimum 0 Tage; Maximum 1089 Tage. (Quelle: GenBIn-Datenbank, RKI)

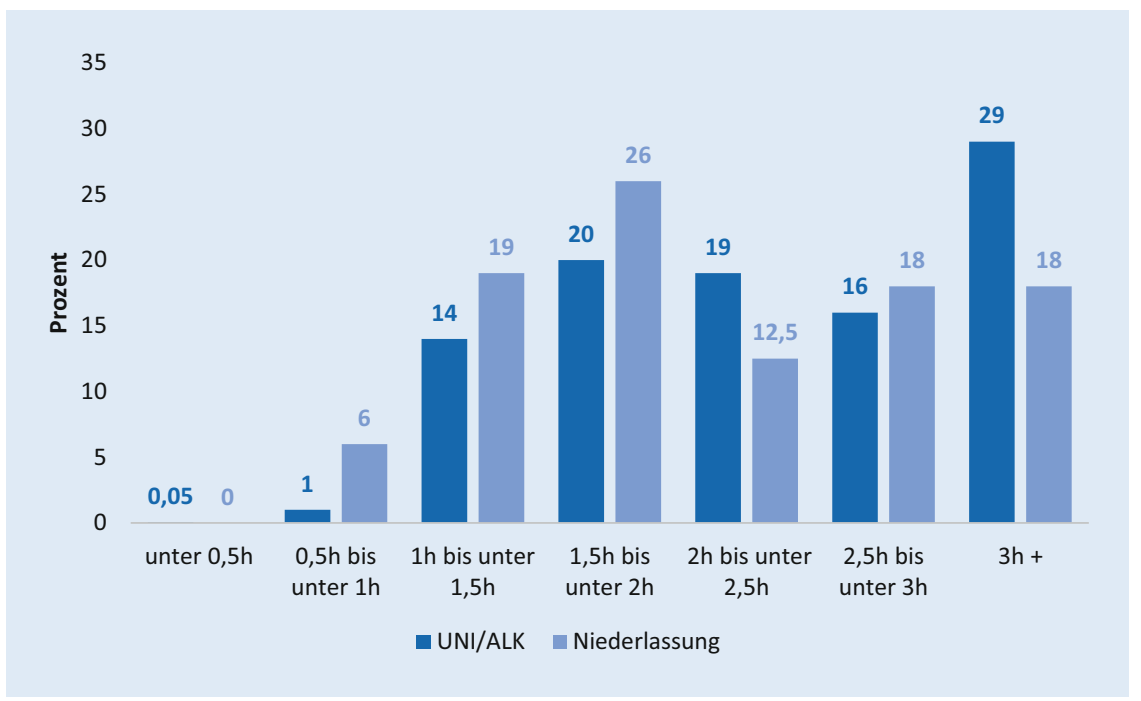

Abb. $5 \Delta$ GenBIn-Datenbank:Dauer des gesamten Zeitaufwandes pro Fall einschließlich Hintergrundarbeit. Nach Beratungseinrichtung in 2016/2017 (\%). GenBIn: Universität/ALK: gültige Fälle 2052, fehlende Fälle 12. Die Dauer der Beratung wurde in einer halbstündigen $(0,5 \mathrm{~h})$ Klassifizierung erhoben: Mittelwert 5,71; Median 5,28; Modus 4; Std.-Abweichung 2,376; Minimum 1, Maximum 25; Niederlassung: gültige Fälle 1092, fehlende Fälle 9; Mittelwert 5,06; Median 4,61; Modus 4,00; Std.-Abweichung 2,329. (Quelle: GenBIn-Datenbank, RKI)

Bestimmung des Risikos autosomaler Trisomien 13, 18 und 21 mittels eines molekulargenetischen Tests (nichtinvasiver Pränataltest [NIPT]) wurden überwiegend in den Beratungseinrichtungen der Niederlassungen $(72 \%)$ erbracht. Insgesamt waren die Beratungsanteile vor NIPT sowohl in der Niederlassung $(8,5 \%)$ als auch in den univer-
Herbst 2020 NIPT als Leistung zulasten der GKV im Rahmen der ärztlichen Schwangerenbetreuung angeboten werden sollte.

\section{Diskussion: Zukünftige Entwick- lung - Kapazitätsengpässe in der genetischen Beratung}

Seit 2005/2006 ist ein konstanter Anstieg genetischer Beratungsleistungen in Deutschland zu beobachten. Es kann spekuliert werden, dass dieser Anstieg in Teilen dem damaligen Technologieschub (dem verstärkten Einsatz genomischer Technologien, darunter Array-CGH) und der zunehmenden Kenntnis genetischer, insbesondere molekulargenetischer Grundlagen von Erkrankungen in anderen medizinischen Fachgruppen geschuldet ist. Der starke Anstieg von 2008 zu 2009 kann möglicherweise auch im Zusammenhang mit dem GenDG gesehen werden. Die gesellschaftliche Diskussion im Vorfeld der Gesetzgebung und die in diesem Gesetz betonte Bedeutung der genetischen Beratung könnten zu einer verstärkten Inanspruchnahme beigetragen haben. Die Zunahme von Selbstüberweisungen (GenBIn-Datenbank) kann als Indikator dafür gewertet werden, dass es zunehmend vorinformierte Personen gibt, die Zugang zu mehr genetischen Informationen mithilfe der genetischen Beratung haben möchten, unter anderem für die Abklärung von Risiken für familiäre Krebserkrankungen. Die veränderten relativen Anteile der Zuweiser zu den genetischen Beratungseinrichtungen in der Niederlassung in 2016/2017 können als ein Indikator hierfür gewertet werden. Damit legen die von uns erhobenen Daten die Vermutung nahe, dass der beobachtbare ständige Anstieg genetischer Beratungsleistungen multifaktoriell bedingt ist und nicht allein auf das GenDG zurückzuführen ist.

In der Humangenetik haben die gestiegenen Beratungsleistungen zu längeren Wartezeiten geführt. Das sich seit 2011 ändernde Spektrum der Überweisungsindikationen erhöht die mittlere Dauer des gesamten Zeitaufwandes/Fall. Während - wie diese Untersuchung zeigt - die Inanspruchnahme der genetischen 
Beratung seit 2009 mit einer durchschnittlichen Rate von ca. $6 \%$ pro Jahr anwächst, bleibt die Zahl der hier wesentlichen Leistungserbringer, der vertragsärztlichen Fachärzte für Humangenetik, nahezu konstant. Deren Zahl schwankte in den Jahren 2013 bis 2018 zwischen 187 und 200, gezählt nach Bedarfsplanungsgewicht (Vollzeitäquivalenten), mit einer nur sehr geringfügig ansteigenden Ten$\operatorname{denz}$ [8]. Es lässt sich aus den hier präsentierten Daten und der Annahme eines ärztlichen Aufwands von 2,5 h pro Beratungsfall [6] grob abschätzen, dass ein Facharzt für Humangenetik derzeit etwa die Hälfte seiner vertragsärztlichen Tätigkeit für die humangenetische $\mathrm{Be}$ ratung einsetzt. Es ist absehbar, dass bei einer Fortsetzung beider Trends der steigenden Inanspruchnahme der humangenetischen Beratung und einer beinahe konstanten Zahl der Leistungserbringer - Kapazitätsgrenzen erreicht werden. Eine nennenswerte Steigerung der Zahl von Fachärzten ist angesichts der Nachwuchslage in diesem Fachgebiet in absehbarer Zeit nicht zu erwarten [9].

Es bleibt abzuwarten, ob und inwieweit die fachgebundene genetische $\mathrm{Be}$ ratung auf der Basis der GEKO-Richtlinie [1] sowie deren Implementierung in die (Muster-)Weiterbildungsordnung einiger Facharztgebiete [10] zu einer vom Gesetzgeber intendierten Verbesserung der Versorgungslage beiträgt. Daten hierzu wären wünschenswert.

\section{Korrespondenzadresse}

Prof. Dr. med. Jörg Schmidtke

Institut für Humangenetik, Medizinische

Hochschule Hannover

Carl-Neuberg-Str. 1, 30685 Hannover,

Deutschland

schmidtke.joerg@mh-hannover.de

Danksagung. Wir danken Frau Ebru Ataseven und Herrn Joachim Heuer (Zentralinstitut der Kassenärztlichen Bundesvereinigung) und den in $\bullet$ Abb. 1 genannten Kassenärztlichen Vereinigungen für die zur Verfügung gestellten Daten.

Funding. Open Access funding provided by Projekt DEAL.

\section{Einhaltung ethischer Richtlinien}

Interessenkonflikt. J. Schmidtke, J.T. Epplen, R. Glaubitz, T. Grimm, R.P. Nippert, H. Tönnies, K. Zerres und I. Nippert geben an, dass kein Interessenkonflikt besteht.

Für diesen Beitrag wurden von den Autoren keine Studien an Menschen oder Tieren durchgeführt. Für die aufgeführten Studien gelten die jeweils dort angegebenen ethischen Richtlinien.

Open Access Dieser Artikel wird unter der Creative Commons Namensnennung 4.0 International Lizenz veröffentlicht, welche die Nutzung, Vervielfältigung, Bearbeitung, Verbreitung und Wiedergabe in jeglichem Medium und Format erlaubt, sofern Sie den/die ursprünglichen Autor(en) und die Quelle ordnungsgemäß nennen, einen Link zur Creative Commons Lizenz beifügen und angeben, ob Änderungen vorgenommen wurden.

Die in diesem Artikel enthaltenen Bilder und sonstiges Drittmaterial unterliegen ebenfalls der genannten Creative Commons Lizenz, sofern sich aus der Abbildungslegende nichts anderes ergibt. Sofern das betreffende Material nicht unter der genannten Creative Commons Lizenz steht und die betreffende Handlung nicht nach gesetzlichen Vorschriften erlaubt ist, ist für die oben aufgeführten Weiterverwendungen des $\mathrm{Ma}$ terials die Einwilligung des jeweiligen Rechteinhabers einzuholen.

Weitere Details zur Lizenz entnehmen Sie bitte der Lizenzinformation auf http://creativecommons.org/ licenses/by/4.0/deed.de.

\section{Literatur}

1. Richtlinie der Gendiagnostik-Kommission (GEKO) über die Anforderungen an die Qualifikation zur und Inhalte der genetischen Beratung gemäß § 23 Abs. 2 Nr. 2a und $\S 23$ Abs. 2 Nr. 3 GenDG. In der Fassung vom 01.07.2011, veröffentlicht und in Kraftgetreten am 11.Juli 2011 (2011). Bundesgesundheitsbl Gesundheitsforsch Gesundheitsschutz 54:1248-1256

2. Varga $O$, Soini $S$, Kääriäinen $H$ et al (2012) Definitions of genetic testing in European legal documents.J Community Genet 3:125-141

3. Soini S (2012) Genetic testing legislation in Western Europe-a fluctuating regulatory target. J Community Genet 3:143-153

4. Murray TH (2019) Is genetic exceptionalism past its sell-by date? On genomic diaries, context, and content. Am J Bioeth 19:13-15

5. Schmidtke J, Pabst B, Nippert I (2005) DNA-based genetic testing is rising steeply in a national health care system with open access to services A survey of genetic test use in Germany, 1996-2002. Genet Test 9:80-84

6. Pabst B, Schmidtke J (2007) Inanspruchnahme humangenetischer Leistungen in Deutschland. In Schmidtke J (Hrsg) Gendiagnostik in Deutschland. Status quo und Problemerkundung. Supplement zum Gentechnologiebericht. Forum W Wissenschaftlicher Verlag, Limburg, S 195-203

7. Tönnies H, Nippert I (2019) Forschungsbericht Genetische Beratung und Inanspruchnahme, RKI Berlin 17.06. 2019. https://www.rki.de/DE/Content/ Kommissionen/GendiagnostikKommission/
GenBIn2/GenBIn2 node.html. Zugegriffen: 25. Aug. 2019 (Förderung Bundesministerium für Gesundheit (BMG); Förderkennzeichen ZMVi12515-FSB-791)

8. Bundesarztregister (2019) https://www.kbv.de/ html/bundesarztregister.php;. Zugegriffen: 25 . Aug. 2019 (jeweils S. Tab. 4.B in den hier archivierten Dokumenten)

9. Scholz C (2018) Ergebnisse einer Umfrage zur Weiterbildungssituation in der Humangenetik in Deutschland. medgen 30:523-529

10. Bundesärztekammer (2018) (Muster-)Weiterbildungsordnung 2018. https://www. bundesaerztekammer.de/fileadmin/user upload/downloads/pdf-Ordner/Weiterbildung/ MWBO-16112018.pdf.Zugegriffen:29. März 2019 